
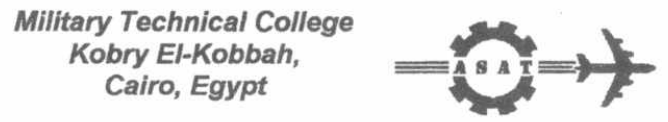

$9^{\text {th }}$ International Conference

On Aerospace Sciences \&

Aviation Technology

\title{
THE DYNAMICS OF VARIABLE EXHAUST NOZZLE LINKAGE MECHANISM OF MILITARY AERO-GAS TURBINE
}

\author{
Suresh Srivastava*, Dibaker Sen ${ }^{\star \star}$, T. S. Mruthyunjaya ${ }^{\star \star} \&$ T. Mohan Rao*
}

\begin{abstract}
The military aero gas turbines of modern day fighter aircraft are fitted with fully variable convergent-divergent (C-D) exhaust nozzle in order to meet the optimum performance requirements of the engine at various operating conditions. The variation in nozzle area is achieved through a flap actuation linkage mechanism that is operated by a set of hydraulic actuators powered by an engine driven integrated hydraulic power pack. This paper presents the methodology developed for dynamic force analysis of an axisymmetric single actuation C-D nozzle mechanism which meets the prescribed schedule of nozzle throat area to exit area ratio as a function of actuator stroke. A computer program has been developed for the dynamic force analysis. One-dimensional isentropic relationships (with throat correction) for flow with area changes are used to compute the pressure distribution across the axisymmetric C-D nozzle. The results of force analysis are the required actuating hydraulic force and the reaction forces of various joints which are used for the stress analysis and mechanical design of individual links as well as for configuring the hydraulic actuation system required for operating the variable exhaust nozzle. An illustrative example has been provided to demonstrate the procedure.
\end{abstract}

Key Words : Nozzle linkage, Gas turbine, Force analysis, Aerodynamic load.

\footnotetext{
* Scientist, Gas Turbine Research Establishment, C. V. Raman Nagar, Bangalore - 560093 , India *t. Department of Mechanical Engg., Indian Institute of Science, Bangalore - 560 012, India
} 


\section{NOMENCLATURE}

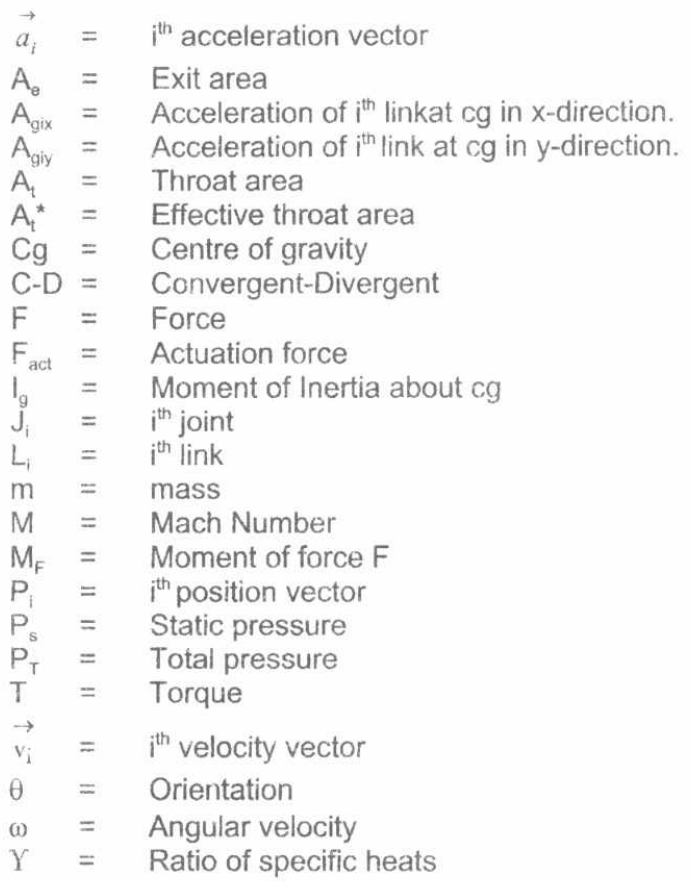

\section{INTRODUCTION}

Aircraft propulsion systems are sized to satisfy critical mission points such as subsonic cruise, high altitude take off, maximum acceleration or high altitude dash for tactical military missions. This sizing determines the inlet and exhaust nozzle areas required through variable geometry at various flight operating conditions.

Variable area nozzles are required for gas turbine engine cycles where large variation in nozzle exit area is necessary. In nearly all cases this area variation is a result of afterburning engine cycles that require area increases of $50-150 \%$ from non afterburning to full afterburning operation ([1], [2]). The Convergent-Divergent (C-D) nozzle is most common with afterburning engines of military fighter aircraft that must achieve supersonic speeds. Supersonic flight speeds result in high engine exhaust nozzle pressure ratios. Use of C-D nozzle enables the engine exhaust gases to be expanded efficiently resulting in an optimum production of engine net thrust.

The sensitivity of engine net thrust to nozzle performance is higher than any other engine component. For this reason, it is extremely important to obtain the highest possible nozzle performance with due consideration for cost, weight, complexity, reliability and maintainability. Development of linkage mechanism for actuation of variable axisymmetric convergent-divergent exhaust nozzle forms one of the high priority technology development tasks for a modern aero gas turbine. A crucial 
problem to be solved here is the dynamic force analysis of the linkage for actuation of the flaps of the variable geometry nozzle. The present work addresses this particular problem. One-dimensional isentropic relationships (with throat correction) for flow with area changes are used to compute the pressure distribution across the axisymmetric C-D nozzle. The static pressures over the flaps are integrated to find the equivalent point gas load and its point of application on the convergent and divergent flaps.

\section{THE C-D NOZZLE LINKAGE}

A cross-sectional schematic of a typical axisymmetric C-D nozzle actuation linkage mechanism is shown in Fig.1. The primary nozzle is actuated by a cam and roller system through mechanically synchronised hydraulic actuators, whereas the secondary nozzle motion is achieved by the linkage system. The C-D nozzle linkage is a one degree of freedom system having 5 links and 6 joints which include 4 revolute joints, 1 prismatic joint and 1 cam-roller joint. A number of convergent (primary) and divergent (secondary) flaps together constitute the C-D nozzle. Each convergent and corresponding divergent flap is actuated by the linkage mechanism. Thus the total number of linkages actuating the C-D nozzle is equal to number of flaps constituting the axisymmetric C-D nozzle.

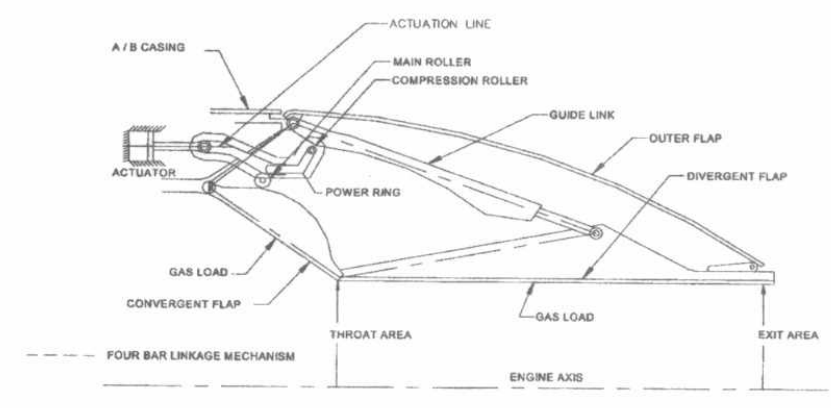

Fig.1. Schematic of C-D Nozzle Linkage

The axisymmetric variable exhaust nozzle of aero gas turbine is assembled to the aft flange of the afterburner casing of aero-gas turbine. There are two cam profiles one on the primary flap and other on the guide link. Main rollers make contact with the cam on the primary flap and compression rollers contact with the cam on the guide link. The Power ring holds main rollers and compression rollers and is connected to the hydraulic actuators. The primary flaps are attached by the hinge pin bolts to the aft inner diameter of the afterburner casing. Assembled to the aft end of primary flaps are secondary flaps. These components are also hinge pinned together. The guide links function as pivot points for the secondary flaps while the variable exhaust nozzle is in operation. Primary flaps constitute the convergent portion of the C-D nozzle and control the throat area. Secondary flaps constitute the divergent portion of the C-D nozzle and control the exit area. Main rollers and compression rollers are constrained to move in an axial direction parallel to the engine axis. 
When the engine is in operation, exhaust gases create either positive (radially outward) or negative (radially inward) force on the convergent and divergent flaps. At a given time, only one of the two cam-roller pairs viz. cam-roller pair on primary flap or cam-roller pair on guide link is in contact and actuates the mechanism. The camroller mechanism should give a linear relationship between the roller stroke and the convergent flap angle. The cam-roller mechanism on the guide link gives the same area-ratio schedule as obtained when the actuation is at the cam-roller pair on the primary flap. The variation in the orientation of the guide link between fully closed and fully open position of the nozzle is non-linear. A positive force holds the cam of primary flaps against the power ring main rollers. A negative force holds the cam of guide link against the compression rollers. As the actuator rod extends or retracts, the nozzle opens or closes through the cam operated flap actuation linkage mechanism.

\section{AERODYNAMIC LOAD PREDICTION}

Nozzle pressure distributions are used to determine hardware structural loads and actuation system loads. These loads form the input for the force analysis of the C-D nozzle linkage and thus effect detailed mechanical design, weight and cost of the C-D nozzle components.

Internal pressure distribution can be estimated for primary and secondary nozzle flaps to cover all nozzle geometries and operating conditions. Pressure distributions can be obtained either from scale model tests or from analytical methods. Scale model tests are more accurate; however, it is not always possible to obtain test data, particularly early in a development program.

Using the following one dimensional isentropic equations the static pressure at every $1 \mathrm{~mm}$ along the convergent flap is evaluated by iterating for subsonic mach number and supersonic mach number respectively $[3,4]$.

$$
\begin{gathered}
\frac{P_{Y}}{P_{S}}=\left(1+\frac{\gamma+1}{2} M^{2}\right)^{\frac{\gamma}{(\gamma-1)}} \\
\frac{A_{t}}{A_{t}{ }^{*}}=\frac{1}{M}\left[\frac{2}{\gamma+1}+\frac{\gamma-1}{\gamma+1} M^{2}\right]^{\frac{\gamma+1}{2(\gamma+1)}}
\end{gathered}
$$

Because of the sharp corner throat, sudden over expansion of the flow immediately down stream of the throat causes pressure distributions to deviate significantly from that predicted by one dimensional analysis. The amount of deviation is dependent on both primary and secondary nozzle half cone angles and the amount of cooling slot flow if present. To incorporate this deviation, throat correction correlation is used [5] .

\section{VELOCITY ANALYSIS}

The velocity analysis of the four bar linkage, comprising of binary link and revolute joints is carried out by Dyad method [6]. Two adjacent binary links with a revolute joint connecting them are considered as shown in the Fig.2. 


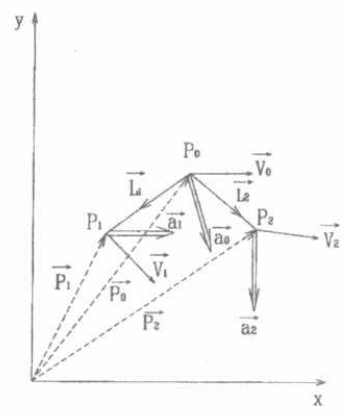

Fig.2. Velocity and acceleration analysis

For given values of velocity vectors $\vec{V}_{1}$ and $\vec{V}_{2}$, position vectors, $\vec{P}_{0}, \vec{P}_{1}$ and $\vec{P}_{2}$ and link vectors $\vec{L}_{1}$ and $\vec{L}_{2}$,

$$
\begin{aligned}
& \hat{L 1}=\frac{1}{L_{1}}\left[\frac{\overrightarrow{P_{01 x}}}{\overrightarrow{P_{01 y}}}\right] \\
& \hat{L} 2=\frac{1}{L_{2}}\left[\frac{\overrightarrow{P_{02 x}}}{\overrightarrow{P_{02 y}}}\right]
\end{aligned}
$$

where $\boldsymbol{P}_{i j}=\boldsymbol{P}_{j}-\boldsymbol{P}_{I}$

From rigidity consideration,

$$
\begin{aligned}
& \vec{V}_{1} \bullet \hat{L_{1}}=\overrightarrow{V_{0}} \bullet \hat{L_{1}} \\
& \vec{V}_{2} \bullet \hat{L_{2}}=\vec{V}_{0} \bullet \hat{L_{2}}
\end{aligned}
$$

Multiplying both sides of equation $(5)$ and $(6)$ by $L 1$ and $L 2$ respectively, we get

$$
\begin{aligned}
& V_{1 x} P_{01 x}+V_{1 y} P_{01 y}=V_{0 x} P_{01 x}+V_{0 y} P_{01 y} \\
& V_{2 x} P_{02 x}+V_{2 y} P_{02 y}=V_{0 x} P_{02 x}+V_{0 y} P_{02 y}
\end{aligned}
$$

Thus the components of velocity at $P_{0}$, where the two binary links $L_{1}$ and $L_{2}$ are connected by revolute joint, is given by the expression

$$
\left[\begin{array}{l}
V_{0 x} \\
V_{0 y}
\end{array}\right]=\left[\begin{array}{ll}
P_{01 x} & P_{01 y} \\
P_{02 x} & P_{02 y}
\end{array}\right]^{-1}\left[\begin{array}{l}
\vec{V}_{1} \cdot \vec{L}_{1} \\
\vec{V}_{2} \bullet \vec{L}_{2}
\end{array}\right]
$$

\section{ACCELERATION ANALYSIS}

The acceleration analysis of the four bar linkage, comprising of binary links and revolute joints, is carried out by Dyad method [6]. Two adjacent binary links with a revolute joint connecting them are considered as shown in Fig. 3. 


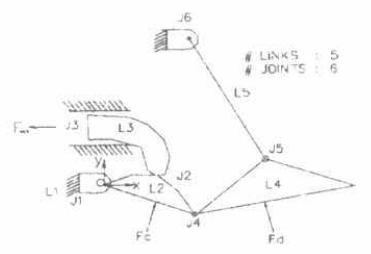

Fig.3.C-D Nozzle linkage with actuation at cam roller pair on primary flap.

Given values of angular velocities $\omega_{1}$ and $\omega_{2}$, acceleration vectors $\overrightarrow{a_{1}}$, and $\overrightarrow{a_{2}}$, position vectors $\vec{P}_{0}, \vec{P}_{1}$ and $\vec{P}_{2}$ and link vectors $\vec{L}_{1}$ and $\vec{L}_{2}$,

From rigidity consideration,

$$
\begin{aligned}
\overrightarrow{a_{0}} \cdot \hat{L}_{1} & =\overrightarrow{a_{1}} \bullet \hat{L_{1}}+\omega_{1}{ }^{2} L_{1} \\
\vec{a}_{0} \bullet \hat{L_{2}} & =\overrightarrow{a_{2}} \bullet \hat{L}_{2}+\omega_{2}{ }^{2} L_{2}
\end{aligned}
$$

Multiplying both sides of equation (10) and (11) by $L_{1}$ and $L_{2}$ respectively, we get

$$
\begin{aligned}
& a_{0 x} P_{01 x}+a_{0 y} P_{01 y}=\vec{a}_{1} \bullet \vec{L}_{1}+\omega_{1}{ }^{2} L_{1}^{2} \\
& a_{0 x} P_{02 x}+a_{0 y} P_{02 y}=\overrightarrow{a_{2}} \bullet \vec{L}_{2}+\omega_{2}{ }^{2} L_{2}^{2}
\end{aligned}
$$

Thus, the components of acceieration at $P_{0}$, where the two binary links $L_{1}$ and $L_{2}$ are connected by revolute joint, are given by the expression

$$
\left[\begin{array}{l}
a_{0 x} \\
a_{0 y}
\end{array}\right]=\left[\begin{array}{ll}
P_{01 x} & P_{01 y} \\
P_{02 x} & P_{02 y}
\end{array}\right]^{-1}\left[\begin{array}{l}
\overrightarrow{a_{1}} \cdot \vec{L}_{1}+\omega_{1}{ }^{2} L_{1}{ }^{2} \\
\overrightarrow{a_{2}} \cdot \overrightarrow{L_{z}}+\omega_{2}{ }^{2} L_{2}{ }^{2}
\end{array}\right]
$$

\section{FORCE ANALYSIS}

Dynamic force analysis of linkage mecharism has been carried out by considering the combined action of all the applied forces, reactive forces and inertia forces simultaneously on various links and joints constituting the mechanism. When the whole system is in equilibrium, every part of the system must also be in equilibrium. During engine operating conditions when the exhaust gas tend to open the nozzle, the linkage actuation is through the can-roller pair on primary flap.

The C-D Nozzle linkage configuration with actuation at cam roller pair on primary fiap is shown in Fig 3. 
The force analysis of the linkage mechanism is obtained by matrix method $[7,8]$. Three independent equations of dynamic equilibrium is written for each moving link in the following manner.

$$
\begin{aligned}
& \Sigma F_{x}=m A_{g x} \\
& \Sigma F_{y}=m A_{g y} \\
& \Sigma T_{g}=I_{g} \alpha_{k}
\end{aligned}
$$

Referring to the C-D Nozzle linkage of Fig.3, dynamic equilibrium equations for the 4 moving links L2, L3, L4, L5 are written as under :

Link 2 :

$$
\begin{gathered}
F_{12 y}+F_{32 y}+F_{42 y}+F_{c x}=m_{2} A_{g 2 x} \\
F_{12 y}+F_{32 y}+F_{42 y}+F_{c y}=m_{2} A_{g 2 y} \\
M_{F_{12 / g}}+M_{F_{32 / g}}+M_{F_{42 / g}}+M_{F_{c / g}}=I_{g 2 \alpha_{2}}
\end{gathered}
$$

Link 3 :

$$
\begin{gathered}
F_{a c t}+F_{23 x}=m_{3} A_{g 3 x} \\
F_{13 y}+F_{23 y}=0 \\
M_{F_{13 / g}}+M_{F_{23 / g}}=0
\end{gathered}
$$

Link 4 :

$$
\begin{gathered}
F_{24 x}+F_{54 x}+F_{d x}=m_{4} A_{g 4 x} \\
F_{24 y}+F_{54 y}+F_{d y}=m_{4} A_{g 4 y} \\
M_{F_{24 / g}}+M_{F_{54 / g}}+M_{F_{d / g}}=I_{g 4} \alpha_{4}
\end{gathered}
$$

Link 5 :

$$
\begin{gathered}
F_{15 x}+F_{45 x}=m_{5} A_{g 5 x} \\
F_{15 y}+F_{45 y}=m_{5} A_{g 5 y} \\
M_{F_{15 / g}}+M_{F_{45 / g}}=I_{g 5} \alpha_{5}
\end{gathered}
$$

The static pressure distribution over the C-D nozzle is integrated to obtain the equivalent point gas loads $\left(F_{c}\right.$ and $\left.F_{d}\right)$ and its corresponding point of application on the convergent and divergent flaps i.e. ' $c$ ' and ' $d$ '.

In above equations, the following expression of the moment of a force around a fulcrum is used.

$$
M_{F}=r_{x} F_{y}-r_{y} F_{x}
$$

This comes from the determinant form of the vector cross product $\mathbf{M}_{\mathrm{F}}=\mathbf{r} \mathbf{x} \mathbf{F}$. Here, $r$ is a vector from the fulcrum to the point of application of the force as shown in Fig.4.

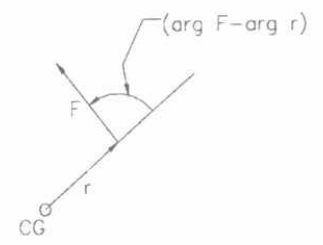

Fig.4. Notation for Moment of a force 
The above system of equations is expressed in matrix form as

where

$$
\left[F_{L}\right]=[L]\left[F_{B}\right]
$$

$\left[F_{U}\right]=$ Column matrix of known external load plus inertia forces and torques.

$[L]=$ Square matrix of known linkage parameters and position angles.

$\left[F_{B}\right]=$ Column matrix of unknown bearing forces and input torque.

The above set of linear equations have been solved for the unknowns using Gauss Jordan Elimination method.

A computer program has been developed for dynamic force analysis of the C-D nozzle linkage. Most of the data, input and derived, used in the force analysis is arranged in two data structure, namely the link data structure and joint data structure.

information:

(1) Link label (2) Number of joints on the link (3) Array of labels of the joints on the link (4) Actuations to the link (5) Mass (6) c.g (7) Moment of inertia about c.g. (8) Velocity of link at c.g. and angular velocity (9) Acceleration of link at c.g. and angular acceleration (10) External force system.

The joint data structure i

information:

(1) Joint label (2) Joint type (3) Array of labels of the labels of the incident link (4) Position/angle depending upon the joint type. For prismatic and cam joint, the angle (radians) represents the line of actuation and the orientation of the common normal at point of contact.

The results of force analysis are the required actuating hydraulic force and the reaction forces of various joints which are used for the stress analysis and mechanical design of individual links as well as for configuring the hydraulic actuation system required for operating the variable exhaust nozzle.

\section{NUMERICAL EXAMPLE}

Consider the following configuration of the mechanism. The joint locations (refer fig 3 ) are as under.

\begin{tabular}{lrll} 
Joint & $\mathbf{x}$ & \multicolumn{1}{r}{$\mathbf{y}$} & Angle \\
J1 & 0.000 & 0.000 & \\
J2 & 0.249 & 0.072 & 0.656 \\
J3 & 0.100 & 0.030 & 0.000 \\
J4 & 0.198 & -0.025 & \\
J5 & 0.400 & 0.006 & \\
J6 & 0.098 & 0.069 &
\end{tabular}


The aerodynamic gas load acting on C-D flaps are as under.

$F_{c x}=9.372, F_{c y}=71.992$, at $c:(0.090,-0.011)$

$F_{d x}=-2.126, F_{d y}=53.577$, at $d:(0.375,-0.018)$

The mass, c.g., moment of inertia and acceleration of the links are as under.

$\begin{array}{lllll} & \text { L2 } & \text { L3 } & \text { L4 } & \text { L5 } \\ \mathbf{x}_{\mathbf{c g}} & 0.097 & 0.088 & 0.434 & 0.150 \\ \mathbf{y}_{\mathbf{c g}} & -0.047 & 0.015 & -0.009 & 0.034 \\ \mathbf{a}_{\mathbf{x}} & 0.003 & 0.000 & 0.050 & 0.000 \\ \mathbf{a}_{\mathbf{y}} & 0.050 & 0.000 & 0.040 & 0.034 \\ \propto & 0.034 & 0.000 & 0.022 & 0.010 \\ \mathbf{m} & 0.020 & 0.020 & 0.042 & 0.032 \\ \mathbf{l} & 0.001 & 0.001 & 0.003 & 0.020\end{array}$

\section{RESULTS}

Input: 127.3415 force units (It) in link 3 w.r.t. 1 JOINT REACTIONS :-

Link 1:

From joint $1<R>$ : force $=7.561$ units @ $8.48^{\circ}$ fromjoint5<R> :force=130.285units @ 168.29 from joint3 $\langle\mathrm{P}\rangle$ : force $=98.017$ units @ $90.00^{\circ}$ moment $=9.259$ units $(\mathrm{ccw})$

Link 2 :

From joint $1<R>$ : force $=7.561$ units @ $188.48^{\circ}$ Fromjoint2 $<\mathrm{C}>$ :force=160.696units@ 217.59 Fromjoint $4<R>$ :force $=128.349$ units @ $12.21^{\circ}$ external(at c):force=72.600units@82.58 at c.g. moment $=-0.866$ units

Link 3 :

Fromjoint2 $<\mathrm{C}>$ : force=160.696 units@ $37.59^{\circ}$ Fromjoint3 $\langle\mathrm{P}\rangle$ : force=98.017units@ $270.00^{\circ}$ moment $=9.259$ units $(\mathrm{cw})$

\section{Link 4 :}

Fromjoint4<R>:force=128.349units@ 192.21 ${ }^{\circ}$ Fromjoint $5<R>$ :force=130.285units@ 348.29 external(at d):force=53.620units@92.27 at c.g moment $=-3.174$ units

\section{Link 5 :}

Fromjoint5<R>:force=130.285units@ 168.29 Fromjoint6<R>:force=130.285units@ 348.29 


\section{CONCLUSION}

In this paper, we have developed the methodology for dynamic force analysis of an axisymmetric singie actuation C-D Nozzle Linkage Mechanism of a military aero gas turbine. The scheme solves the actuation forces and joint reactions by building up the equilibrium equations in a systematic manner. External loads on the nozzle are generally much smaller than the internal loads and has not been considered. Also, the friction at various joints has not been taken into account in the analysis. It can be taken up as a next step to this work.

\section{REFERENCES}

[1] David M. S and Richard R. C , "Thrust performance of a Variable-Geometry Exhaust nozzle of Turbojet", NASA Technical paper 2171, (1983).

[2] Chepkin V., Andreyev A, "The Features of the first furbojet engines with vector controlled nozzles" $35^{\text {th }}$ AIAAASME Joint propulsion conference, AIAA 992672, June (1999).

[3] Cohen \& Rogers,"Gas Turbine Theory", John Wiley \& Sons Inc, New York, (1996).

[4] G.C. Oates "Aircraft propulsion systems", AlAA Education Series, (1994).

[5] Summerfield $M$, "Flow separation in over expanded supersonic Exhaust Nozzles", J. of Jet Propulsion, pp 88 - 96, Oct. (1974).

[6] Suh C. H. \& Radcliffe C.W. "Kinematics and Mechanism Design", John Wiley \& sons, New York, (1978).

[7] Arthor, G. Erdman and Sandor, "Mechanism Design-Analysis and Synthesis", Prentice Hall, Englewood Cliffs, New Jersey, (1990).

[8] Sen D., Dasgupta B. and Mruthyanjaya T.S. "A General scheme for the Dynamic force analysis of planar mechanisms", Proc. Ninth World Congress on the Theory of Machines and Mechanisms-IFTOMM'95, Milan, vol.2, PP894-898, (1995). 